\title{
Comprehensive Diagnosis of Bacterial Infection Associated with Acute Cholecystitis Using Metagenomic Approach
}

\section{OPEN ACCESS}

Edited by:

Fumito Maruyama,

Kyoto University, Japan

Reviewed by:

Gabriel Sandblom,

Karolinska Institutet, Sweden

Shashank Gupta,

Brown University, USA

*Correspondence:

Manabu Kujiraoka

manabu.kujiraoka@med.toho-u.ac.jp

Specialty section:

This article was submitted to

Infectious Diseases,

a section of the journal

Frontiers in Microbiology

Received: 18 January 2017 Accepted: 04 April 2017

Published: 20 April 2017

Citation:

Kujiraoka M, Kuroda M, Asai K,

Sekizuka T, Kato K, Watanabe M, Matsukiyo H, Saito T, Ishii T, Katada N,

Saida Y and Kusachi S (2017) Comprehensive Diagnosis of Bacterial

Infection Associated with Acute Cholecystitis Using Metagenomic Approach. Front. Microbiol. 8:685.

doi: 10.3389/fmich.2017.00685

\begin{abstract}
Manabu Kujiraoka ${ }^{1,2 *}$, Makoto Kuroda ${ }^{2}$, Koji Asai ${ }^{1}$, Tsuyoshi Sekizuka ${ }^{2}$, Kengo Kato ${ }^{2}$, Manabu Watanabe ${ }^{1}$, Hiroshi Matsukiyo ${ }^{1}$, Tomoaki Saito ${ }^{1}$, Tomotaka Ishii ${ }^{1}$, Natsuya Katada ${ }^{1}$, Yoshihisa Saida ${ }^{1}$ and Shinya Kusachi ${ }^{1}$

${ }^{1}$ Department of Surgery, Toho University Ohashi Medical Center, Tokyo, Japan, ${ }^{2}$ Laboratory of Bacterial Genomics, Pathogen Genomics Center, National Institute of Infectious Diseases, Tokyo, Japan
\end{abstract}

Acute cholecystitis (AC), which is strongly associated with retrograde bacterial infection, is an inflammatory disease that can be fatal if inappropriately treated. Currently, bacterial culture testing, which is basically recommended to detect the etiological agent, is a time-consuming (4-6 days), non-comprehensive approach. To rapidly detect a potential pathogen and predict its antimicrobial susceptibility, we undertook a metagenomic approach to characterize the bacterial infection associated with AC. Six patients (P1-P6) who underwent cholecystectomy for AC were enrolled in this study. Metagenome analysis demonstrated possible single or multiple bacterial infections in four patients (P1, P2, P3, and P4) with 24-h experimental procedures; in addition, the CTX-M extended-spectrum B-lactamase (ESBL) gene was identified in two bile samples ( $P$ 1 and P4). Further whole genome sequencing of Escherichia coli isolates suggested that CTX-M-27-producing ST131 and CTX-M-14-producing novel-ST were identified in P1 and P4, respectively. Metagenome analysis of feces and saliva also suggested some imbalance in the microbiota for more comprehensive assessment of patients with AC. In conclusion, metagenome analysis was useful for rapid bacterial diagnostics, including assessing potential antimicrobial susceptibility, in patients with AC.

Keywords: acute cholecystitis, metagenome analysis, next-generation sequencing, bacteriological analysis, gut microbiota

\section{INTRODUCTION}

Acute cholecystitis (AC) is the acute inflammation of the gallbladder. AC is usually associated with cholelithiasis, with gallstones detected in $>90 \%$ cases. Additional causes include circulation defect of the gallbladder wall, bacterial infection, and chemical disorder (van der Linden and Sunzel, 1970; Wang et al., 2016). The Tokyo Guidelines 07, published in 2007 following the international consensus meeting on the management of AC and cholangitis, were revised in 2013 (TG13)

Abbreviations: AC, Acute cholecystitis; ERCP, Endoscopic retrograde cholangiopancreatography; ESBLs, Extended-spectrum ß-lactamase; IBD, Inflammatory bowel disease; MLST, Multilocus sequence typing; NA, Not applicable; NGS, Next-generation sequencing; TG13, Tokyo guidelines 2013. 
(Takada et al., 2007, 2013). The guidelines recommend treatment based on the severity, with patients generally treated with antimicrobial agents before operation, drainage, or general care is considered. When conservative treatment with drainage is selected, antimicrobial agents are administered depending on the bile culture examination and antimicrobial susceptibility testing results.

Asai et al. reported that bactibilia was detected in 96 of 136 patients with AC (58.6\%) (Asai et al., 2012), whereas Csendes et al. reported the rate, including that of cholelithiasis, was 22$46 \%$ (Csendes et al., 1996). The rate of bactibilia was reported to be $72 \%$ in TG13 (Takada et al., 2013); hence, the guidelines recommend bile culture for all cases. However, bile culture is a time-consuming, non-comprehensive approach that requires several days to obtain the results. Delay of appropriate treatment might result in a fatal condition and might increase antimicrobial resistance. Hence, the establishment of rapid diagnostics is important to determine an appropriate treatment. Furthermore, the microflora of the gut is believed to be associated with the prevention of infection and fatal condition (Wells et al., 2000; Wu et al., 2013; Liu et al., 2015). Currently, there are no reports regarding the use of a metagenomic approach to reveal comprehensive microbiota analysis of patients with AC.

Metagenome analysis by next-generation DNA sequencing (NGS) has led to a new method of identifying the etiological agents of an infectious disease (Tang and Chiu, 2010; Chan et al., 2012). By directly sequencing millions of DNA/RNA molecules in a specimen and matching the sequences to those in a database, pathogens can be inferred (Takeuchi et al., 2014). The conventional method requires bacteria to be individually cultured, whereas metagenome analysis is a comprehensive approach that enables the direct sequencing of bacterial DNA without bacterial cultivation (Takeuchi et al., 2014).

Here, we report the results of metagenome analysis of six cases (P1-P6) who underwent cholecystectomy for AC. This study was expected to provide additional information, such as bacterial genotyping and ratios of bacterial species compared with that provided by conventional examination. The risk factors of AC, which is associated with gut microbiota, may also become gradually clear with this approach. Moreover, the possibilities of obtaining rapid genotype analysis results of potential pathogens and also rapid evaluation of antimicrobial resistance are promising aspects of this technique.

\section{MATERIALS AND METHODS}

\section{Patients}

Between May 2015 and March 2016, six patients (P1-P6) who underwent cholecystectomy for AC at the Department of Surgery, Toho University Ohashi Medical Center were enrolled in this study. Written informed consent was obtained from the participants. The patients were diagnosed as having AC and were performed recommended antimicrobial treatment based on the TG13 severity grade during the waiting period for operation. The study protocol was approved by the ethics committee of the Toho University Ohashi medical Center (Approval No. 14-58, $11 / 18 / 2014$ ) and National Institute of Infectious Diseases in Japan
(Approval No. 642, 12/11/2015). It was conducted according to the principles of the Declaration of Helsinki.

\section{Materials}

Bile samples were aseptically collected during the operation and divided into three tubes (two aerobic porters and one anaerobic porter, Kenki porter ${ }^{\circledR}$ ). One aerobic and one anaerobic porter immediately underwent conventional culture and antimicrobial susceptibility testing at our hospital. The other aerobic porter was immediately frozen at $-20^{\circ} \mathrm{C}$ for future metagenome analysis. In addition, the patients put saliva and feces into an aerobic porter during hospitalization, which was also immediately frozen at $-20^{\circ} \mathrm{C}$.

\section{Antimicrobial Susceptibility Testing}

Antimicrobial susceptibility testing was performed using Neg EN Combo $1 \mathrm{~T}^{\circledR}$ panels (Microscan Walkaway 96SI: Siemens) on the basis of the Clinical and Laboratory Standards Institute criteria (M100-S22) (Clinical and Laboratory Standards Institute, 2012). Extended-spectrum ß-lactamases (ESBLs) were assessed by a disk-diffusion test using cefotaxime, ceftazidime, and cefpodoxime with or without clavulanate.

\section{Metagenome Analysis}

The clinical specimens were mixed with EDTA (final $10 \mathrm{mM}$ ), SDS (final 1\%), and proteinase $\mathrm{K}$ (final $100 \mu \mathrm{g} / \mathrm{ml}$ ) and incubated at $50^{\circ} \mathrm{C}$ for $30 \mathrm{~min}$. The samples were treated with an equal volume of 0.1 -mm-diameter glass beads and lysed by bead beating using a vortex at a maximum speed for $10 \mathrm{~min}$ with a Genie2 vortex adapter (MO BIO). Following centrifugation to remove the debris, the supernatants were extracted using the QIAGEN QIAquick PCR Purification Kit. A DNA-seq library was prepared using the Illumina Nextra ${ }^{\circledR}$ XT DNA Sample Preparation Kit (Illumina; San Diego, CA, USA) and the indexing method. A deep sequencing run for single-end short reads (150mer) using the MiSeq reagents kit v3 was performed using a MiSeq sequencer (Illumina; San Diego, CA, USA).

\section{Bioinformatics}

To identify potential pathogens and detect antimicrobial resistance genes, sequencing reads were analyzed by the MePIC2 (Takeuchi et al., 2014), Krona (Ondov et al., 2011), and MEGAN6 software (Huson et al., 2007, 2016). To identify the bacterial sequence type and antimicrobial resistance, sequencing reads were analyzed by multilocus sequence typing (MLST) and Resfinder, respectively (Larsen et al., 2012; Zankari et al., 2012).

\section{Nucleotide Sequence Accession Number}

The metagenome analysis short-read sequences have been deposited in the DNA Data Bank of Japan (accession numbers: DRA005134).

\section{RESULTS}

The clinical presentations of all six patients are shown in Table $\mathbf{1 .}$ All patients underwent cholecystectomy for AC. The median 
TABLE 1 | Clinical presentations and results of bacterial cultures.

\begin{tabular}{|c|c|c|c|c|c|c|}
\hline Patient ID & P1 & P2 & P3 & P4 & P5 & P6 \\
\hline \multicolumn{7}{|l|}{ CHARACTERISTICS OF PATIENTS } \\
\hline Age (years) & 85 & 78 & 76 & 53 & 43 & 44 \\
\hline $\operatorname{Sex}(M / F)$ & $\mathrm{F}$ & M & $\mathrm{M}$ & $\mathrm{F}$ & $\mathrm{M}$ & $\mathrm{F}$ \\
\hline \multirow[t]{2}{*}{ Past medical history } & Angina pectoris & Pleurisy & Lacunar infarction & Chronic pancreatitis & - & Cervical cancer \\
\hline & Rheumatoid arthritis & & Hypertension & & & \\
\hline Cause of acute cholecystitis & Gallbladder stone & Gallbladder stone & Gallbladder stone & Gallbladder stone & - & - \\
\hline \multicolumn{7}{|l|}{ LABORATORY DATA } \\
\hline White blood cell count (cells/mm³) & 10,500 & 14,200 & 10,400 & 5,600 & 12,600 & 3,100 \\
\hline C-reactive protein (mg/dL) & 19.69 & 2.15 & 9.49 & 0.37 & 1.58 & 9.62 \\
\hline \multicolumn{7}{|l|}{ PREOPERATIVE CHARACTERISTICS } \\
\hline Time from onset to operation (h) & 55 & 41 & 46 & 6.4 & 33 & 53 \\
\hline Time from admission to operation (h) & 22 & 41 & 42 & 0.4 & 20 & 39 \\
\hline Drainage & PTGBA & ERCP & NA & NA & NA & NA \\
\hline Antimicrobial agents & CTRX & SBT/CPZ & SBT/CPZ & NA & SBT/CPZ & CTRX \\
\hline Hospitalization (days) & 22 & 10 & 10 & 3 & 4 & 7 \\
\hline \multicolumn{7}{|l|}{ TG 13 SEVERITY CLASSIFICATION } \\
\hline Mild/Moderate/Severe & Mild & Mild & Moderate & Mild & Mild & Moderate \\
\hline \multirow[t]{3}{*}{ Bacterial profile of bile culture } & Escherichia coli (ESBL) & Escherichia coli & Escherichia coli & Escherichia coli (ESBL) & Negative & Negative \\
\hline & & & Klebsiella pneumoniae & & & \\
\hline & & & $\alpha$-hemolytic Streptococcus & & & \\
\hline
\end{tabular}

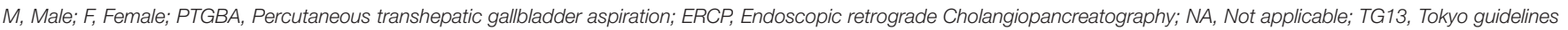
2013; ESBL, Extended-spectrum B-lactamase; CTRX, Ceftriaxone, SBT, Sulbactam, CPZ, Cefoperazone.

age was 64.5 years (range: $43-85$ years) and three were males and three were females. The causes of AC included gallbladder stone obstruction in four cases and unknown in two cases. The median time from onset to operation was $39.5 \mathrm{~h}$, with the median time from admission to operation being $31 \mathrm{~h}$. The patients underwent antimicrobial treatment several times before the operation, except for one case. Conventional bile culture examination results revealed that four cases (P1-P4) were bacteriologically positive and two (P5, P6) were negative (Table 1).

When evaluating mucous membrane inflammation, human DNA sequences were excluded from the MEPIC2 software analysis for ethical reasons, allowing for the detection of pathogens as shown in Figure 1. After this process, DNA derived from Homo sapiens was more abundant in bacteriologically positive cases compared with that in negative cases, suggesting an association between bacterial infection and exfoliation of the gallbladder mucous membrane. The ratio of bacterial DNA was also high in positive cases (Figure 1). Bacterial infection of the gallbladder was rapidly diagnosed by the metagenome approach (within $24 \mathrm{~h}$ ) compared with that by the conventional approach (within 4-6 days). In feces and saliva samples, characteristic bacterial populations were identified, especially in the positive cases. Each case is described in detail.

\section{Patient 1 (P1)}

Only ESBL-positive E. coli was detected in the bile by bacterial culture and antimicrobial susceptibility testing (Table 1). This patient had postoperative complications, such as an intraabdominal abscess.

As shown in Figure 1, the purulent bile was identified to be bacteriologically positive by the metagenomics approach. The ratio of bacterial DNA in the specimen was high, and the etiological agent was E. coli ST131 with the CTX-M-27 gene.

Bacterial detection from feces and saliva samples are shown in Figures 2A,B. Intriguingly, E. coli was detected at a high ratio in feces (60\%) and saliva (17\%). ST131 was also detected in both samples and also in the bile sample. Although E. coli was generally detected in a smaller percentage in feces and saliva (Becker et al., 2015), this result suggested that it was a marked etiological agent of $\mathrm{AC}$ and that dysbiosis may be related to postoperative complications.

\section{Patient 2 (P2)}

During the treatment of pleurisy with levofloxacin, P2 developed AC because of cholelithiasis. LVFX-resistant E. coli was detected by conventional antimicrobial susceptibility testing; however, ESBL was not detected in the bile samples (Table 1). As shown in Figure 1, mild cholestasis was detected in the bile sample, and bactibilia was identified by metagenome analysis. The etiological agent was E. coli. Although, it is unclear whether Enterococcus gallinarum and E. casseliflavus were associated with AC, these were significantly detected by metagenome analysis.

Bifidobacterium was abundantly detected at $68 \%$ in feces (Figure 2A), which is higher than generally detected in a healthy 

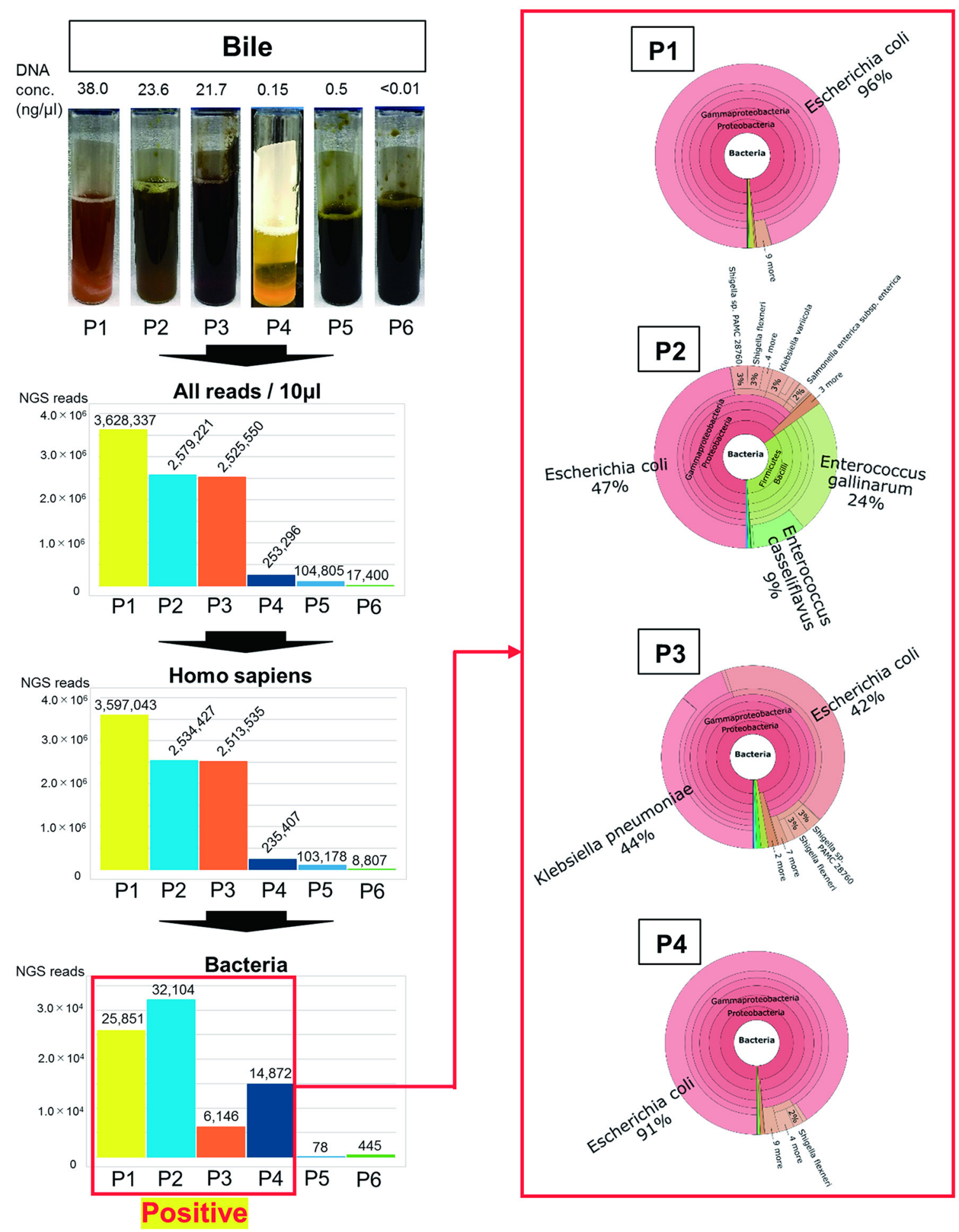

FIGURE 1 | Metagenome analysis of bile specimens. The bile appearance was purulent (P1), mild cholestasis (P2), white (P4), and cholestasis (P3, P5, P6). From metagenome analysis, the ratio of $\mathrm{H}$. sapiens DNA included in the specimens was high for bacteriologically positive cases. Furthermore, a high ratio of bacterial DNA was detected from samples that were bacteriologically positive by conventional culture examinations. The numbers on the bar graph represent the sequencing reads for each patient. Regarding the detected bacteria, the ratios of etiological agents of AC were rapidly identified within $24 \mathrm{~h}$ (shown in red box). 

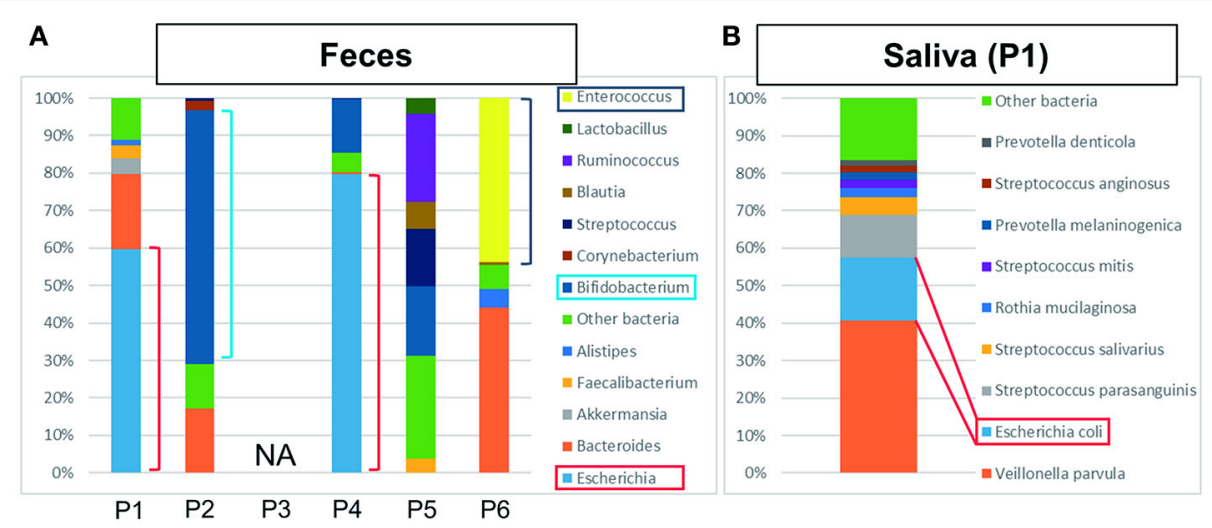

FIGURE 2 | The results of metagenome analysis of feces and saliva. (A) P1-P4 were bacteriologically positive cases, P3 was not applicable (NA) and P5 and P6 were negative cases. In the cases of bactibilia, bacterial turbulence of feces was identified. Especially, a high ratio of E. coli was detected in P1 and P4 suggesting the AC was caused by E. coli. Bifidobacterium was detected at high ratio in P2. The bacterial flora in P5 was dominated by Ruminococcus, Bifidobacterium,

Streptococcus, and "other bacteria." In P6, dysbiosis, determined by the increased amount of Enterococcus detected, may be due to the influence of chemotherapy. (B) Characteristic saliva analysis shown for $\mathrm{P} 1$. A high ratio of $E$. coli was detected.

gut (Odamaki et al., 2016). It was unknown whether P2 received probiotics.

\section{Patient 3 (P3)}

In this case, E. coli, K. pneumoniae, and $\alpha$-hemolytic Streptococcus were detected by the bacterial culture of bile, along with cholestasis (Table 1 and Figure 1). Metagenome analysis suggested that the bile sample was bacteriologically positive for E. coli and K. pneumonia, indicating a diagnosis of AC with multi-infection. We were unable to obtain saliva and feces samples from this patient.

\section{Patient 4 (P4)}

ESBL-positive E. coli was detected by bile culture (Table 1). The appearance of bile was white, and it was bacteriologically positive by metagenome analysis (Figure 1). The etiological agent was E. coli that had the CTX-M-14 gene but with a novel sequence type. E. coli was detected at a high ratio (76\%) in feces by metagenome analysis (Figure 2A). The microbiota of saliva is not significantly different from commensal oral microbiota, which is dominated by Actinobacteria and Streptococcus spp. (Lloyd-Price et al., 2016).

\section{Patient 5 (P5)}

No bacteria were detected by bile culture in this case (Table 1). As shown in Figure 1, the appearance of bile was with cholestasis. Metagenome analysis was negative for bacterial infection, indicating that treatment with antimicrobial agents was not necessary. No significant differential flora was detected in feces (Figure 2A) and saliva. The bacteria detected in the feces appeared to be normal because it was dominated by Ruminococcus (23\%), Bifidobacterium (18\%), Streptococcus (15\%), and "others" that included over two hundreds types of bacterial genera. A lesser amount of Proteobacteria, including E. coli (Qin et al., 2010; Becker et al., 2015), was detected.

\section{Patient 6 (P6)}

The bile culture results were negative (Table $\mathbf{1}$ ). The appearance of bile was with cholestasis (Figure 1). Limited bacterial DNA was detected from the bile by metagenome analysis, similar to P5, suggesting bacteriologically negative samples. As for P5, it is not necessary to treat with antimicrobial agents. The ratio of bacterial species in the feces of the patient was different from that of healthy people, with E. faecium (44\%) significantly detected. This result may be affected by chemotherapy.

The results of metagenome analysis were consistent with those of conventional culture examination and antimicrobial susceptibility testing for all six patients. Although qualitative evaluation was possible by conventional examination, quantitative evaluation was impossible. Metagenome analysis enables the quantitative evaluation. Metagenome analysis has no concern about missing the detection of potential pathogens with unculturable etiological agents.

In addition to the bile samples, feces and saliva samples were analyzed by metagenome approach to provide a more complete assessment of patients with AC. The bacteriologically positive cases showed some imbalance in the microbiota compared with negative cases.

\section{DISCUSSION}

NGS technologies are a new class of sequencing platforms, which differ from previous sequencing methods mainly based on Sanger sequencing, which have been developed and established over the last decade. These new instruments have revolutionized the field of genomics, providing enormous high-throughput and speed, with a wide range of applications now accessible to most laboratories. This technology has been applied to investigate the etiological agent of other inflammatory diseases, such as inflammatory bowel disease (IBD) (Morgan et al., 2012; Dalal and Chang, 2014). Furthermore, high-throughput sequencing has enabled the identification of previously uncharacterized viruses 
or bacteria as the etiological agents of infectious diseases (Tang and Chiu, 2010; Barzon et al., 2011; Chan et al., 2012; Lavezzo et al., 2013; Faria et al., 2016).

In this study, we analyzed six patients with AC using metagenome analysis with NGS. For the case of bactibilia, the metagenome analysis results were consistent with those of conventional culture examination and antimicrobial susceptibility testing.

A greater number of ESBL-positive E. coli NGS reads were detected by metagenome analysis in $\mathrm{P} 1$ and $\mathrm{P} 4$ compared with that in other patients. Several reports have been published on the gut microbiota in patients with IBD showing an increase in the number of species belonging to Proteobacteria, including $E$. coli (Frank et al., 2007; Morgan et al., 2012). The proportion of Proteobacteria in healthy human intestinal microbiota was shown to be $1 \%$ (Tap et al., 2009). AC for P1, who had more $E$. coli in the feces sample compared with healthy people, was most likely caused by E. coli. Furthermore, E. coli was more significantly detected in P1 without vomiting than in healthy people (Clemente et al., 2012). In this case, the postoperative complication of an intraabdominal abscess was detected, prolonging the duration of hospitalization compared with that in other patients (Table 1).

E. coli ST131, which is a particularly virulent strain of E. coli, was first described in (Nicolas-Chanoine et al., 2008). The dissemination has been globally reported, both in the healthcare and community settings (Rogers et al., 2011; Nicolas-Chanoine et al., 2014), and is mostly associated with ESBL production (Banerjee and Johnson, 2014; Nicolas-Chanoine et al., 2014). The detection of E. coli ST131 in all specimens of P1 was pivotal information for the assessment of AC in this patient. Hence, fecal and saliva samples may be used to detect antimicrobial resistance agents for appropriate treatment.

CTX-M-27 and CTX-M-14 genes detected in this study belong to the group of CTX-M-9 (Bonnet, 2004), which is the predominate type in Japan, especially in nosocomial infections (Hawkey and Jones, 2009; Matsumura et al., 2015; Shibasaki et al., 2016). When any infections occur in the hospital, the analysis of CTX-M gene is important for the detection of the bacterial

\section{REFERENCES}

Asai, K., Watanabe, M., Kusachi, S., Tanaka, H., Matsukiyo, H., Osawa, A., et al. (2012). Bacteriological analysis of bile in acute cholecystitis according to the Tokyo guidelines. J. Hepatobiliary Pancreat. Sci. 19, 476-486. doi: 10.1007/s00534-011-0463-9

Banerjee, R., and Johnson, J. R. (2014). A new clone sweeps clean: the enigmatic emergence of Escherichia coli sequence type 131. Antimicrob. Agents Chemother. 58, 4997-5004. doi: 10.1128/AAC.02824-14

Barzon, L., Lavezzo, E., Militello, V., Toppo, S., and Palu, G. (2011). Applications of next-generation sequencing technologies to diagnostic virology. Int. J. Mol. Sci. 12, 7861-7884. doi: 10.3390/ijms12117861

Becker, C., Neurath, M. F., and Wirtz, S. (2015). The intestinal microbiota in inflammatory bowel disease. ILAR J. 56, 192-204. doi: 10.1093/ilar/ilv030

Bonnet, R. (2004). Growing group of extended-spectrum $\beta$-lactamases: the CTX-M enzymes. Antimicrob. Agents Chemother. 48, 1-14. doi: 10.1128/AAC.48.1.1-14.2004 origin and route of transmission. We were unable to identify whether the cases were nosocomial infections because they were all emergency surgery cases. However, both P1 and P4 possibly carried ESBL-producing E. coli.

In this study, we retrospectively performed metagenome analysis on bile, feces, and saliva samples from six AC patients. This approach has been applied to various fields and is expected to be one of the most important diagnostic techniques to treat AC. Indeed, cases of AC where surgical intervention is undecided, the treatment process can be rapidly determined by metagenome analysis of bile collected by percutaneous transhepatic gallbladder aspiration. Hence, NGS technology provides a useful approach for the rapid diagnosis of patients with AC.

\section{AUTHOR CONTRIBUTIONS}

$\mathrm{MnK}, \mathrm{MkK}$, and $\mathrm{KA}$ designed the study concept, revised the manuscript regarding intellectual content, and analyzed the data. TyS and KK provided technical support. MW, HM, TmS, and TI collected the clinical specimens from patients with AC. NK, YS, and SK approved the final draft of this manuscript to be published.

\section{FUNDING}

This study was supported by a Research Program on Emerging and Re-emerging Infectious Diseases from the Japan Agency for Medical Research and Development (grant numbers: $16 \mathrm{fk} 0108119 \mathrm{j} 0001$ and $16 \mathrm{fk} 0108305 \mathrm{j} 0003)$. The funders had no role in the study design, data collection and analysis, decision to publish, or preparation of the manuscript. This study was also supported by JSPS KAKENHI Grant Number 15K10198 and Toho University Project Research Grant Number 27-18.

\section{ACKNOWLEDGMENTS}

The authors would like to thank Enago (http://www.enago.jp) for the English language review.

Chan, J. Z., Pallen, M. J., Oppenheim, B., and Constantinidou, C. (2012). Genome sequencing in clinical microbiology. Nat. Biotechnol. 30, 1068-1071. doi: $10.1038 /$ nbt. 2410

Clemente, J. C., Ursell, L. K., Parfrey, L. W., and Knight, R. (2012). The impact of the gut microbiota on human health: an integrative view. Cell 148, 1258-1270. doi: $10.1016 /$ j.cell.2012.01.035

Clinical and Laboratory Standards Institute (2012). Performance Standards for Antimicrobial Susceptibility Testing; Twenty-second Informational Supplement. Wayne, PA: Clinical and Laboratory Standards Institute.

Csendes, A., Burdiles, P., Maluenda, F., Diaz, J. C., Csendes, P., and Mitru, N. (1996). Simultaneous bacteriologic assessment of bile from gallbladder and common bile duct in control subjects and patients with gallstones and common duct stones. Arch Surg. 131, 389-394. doi: 10.1001/archsurg.1996.01430160047008

Dalal, S. R., and Chang, E. B. (2014). The microbial basis of inflammatory bowel diseases. J. Clin. Invest. 124, 4190-4196. doi: 10.1172/JCI72330 
Faria, N. R., Azevedo Rdo, S., Kraemer, M. U., Souza, R., Cunha, M. S., Hill, S. C., et al. (2016). Zika virus in the Americas: early epidemiological and genetic findings. Science 352, 345-349. doi: 10.1126/science.aaf5036

Frank, D. N., St. Amand, A. L., Feldman, R. A., Boedeker, E. C., Harpaz, N., and Pace, N. R. (2007). Molecular-phylogenetic characterization of microbial community imbalances in human inflammatory bowel diseases. Proc. Natl. Acad. Sci. U.S.A. 104, 13780-13785. doi: 10.1073/pnas.07066 25104

Hawkey, P. M., and Jones, A. M. (2009). The changing epidemiology of resistance. J. Antimicrob. Chemother. 64(Suppl. 1), i3-10. doi: 10.1093/jac/dkp256

Huson, D. H., Auch, A. F., Qi, J., and Schuster, S. C. (2007). MEGAN analysis of metagenomic data. Genome Res. 17, 377-386. doi: 10.1101/gr.5969107

Huson, D. H., Beier, S., Flade, I., Gorska, A., El-Hadidi, M., Mitra, S., et al. (2016). MEGAN community edition-interactive exploration and analysis of large-scale microbiome sequencing data. PLoS Comput. Biol. 12:e1004957. doi: 10.1371/journal.pcbi.1004957

Larsen, M. V., Cosentino, S., Rasmussen, S., Friis, C., Hasman, H., Marvig, R. L., et al. (2012). Multilocus sequence typing of total-genome-sequenced bacteria. J. Clin. Microbiol. 50, 1355-1361. doi: 10.1128/JCM.06094-11

Lavezzo, E., Toppo, S., Franchin, E., Di Camillo, B., Finotello, F., Falda, M., et al. (2013). Genomic comparative analysis and gene function prediction in infectious diseases: application to the investigation of a meningitis outbreak. BMC Infect. Dis. 13:554. doi: 10.1186/1471-2334-13-554

Liu, J., Yan, Q., Luo, F., Shang, D., Wu, D., Zhang, H., et al. (2015). Acute cholecystitis associated with infection of Enterobacteriaceae from gut microbiota. Clin. Microbiol. Infect. 21, 851.e1-e9. doi: 10.1016/j.cmi.2015.05.017

Lloyd-Price, J., Abu-Ali, G., and Huttenhower, C. (2016). The healthy human microbiome. Genome Med. 8:51. doi: 10.1186/s13073-016-0307-y

Matsumura, Y., Johnson, J. R., Yamamoto, M., Nagao, M., Tanaka, M., Takakura, S., et al. (2015). CTX-M-27- and CTX-M-14-producing, ciprofloxacinresistant Escherichia coli of the H30 subclonal group within ST131 drive a Japanese regional ESBL epidemic. J. Antimicrob. Chemother. 70, 1639-1649. doi: $10.1093 / \mathrm{jac} / \mathrm{dkv} 017$

Morgan, X. C., Tickle, T. L., Sokol, H., Gevers, D., Devaney, K. L., Ward, D. V., et al. (2012). Dysfunction of the intestinal microbiome in inflammatory bowel disease and treatment. Genome Biol. 13:R79. doi: 10.1186/gb-2012-13-9-r79

Nicolas-Chanoine, M. H., Bertrand, X., and Madec, J. Y. (2014). Escherichia coli ST131, an intriguing clonal group. Clin. Microbiol. Rev. 27, 543-574. doi: 10.1128/CMR.00125-13

Nicolas-Chanoine, M. H., Blanco, J., Leflon-Guibout, V., Demarty, R., Alonso, M. P., Canica, M. M., et al. (2008). Intercontinental emergence of Escherichia coli clone O25:H4-ST131 producing CTX-M-15. J. Antimicrob. Chemother. 61, 273-281. doi: 10.1093/jac/dkm464

Odamaki, T., Kato, K., Sugahara, H., Hashikura, N., Takahashi, S., Xiao, J. Z., et al. (2016). Age-related changes in gut microbiota composition from newborn to centenarian: a cross-sectional study. BMC Microbiol. 16:90. doi: 10.1186/s12866-016-0708-5

Ondov, B. D., Bergman, N. H., and Phillippy, A. M. (2011). Interactive metagenomic visualization in a Web browser. BMC Bioinformatics 12:385. doi: 10.1186/1471-2105-12-385

Qin, J., Li, R., Raes, J., Arumugam, M., Burgdorf, K. S., Manichanh, C., et al. (2010). A human gut microbial gene catalogue established by metagenomic sequencing. Nature 464, 59-65. doi: 10.1038/nature08821
Rogers, B. A., Sidjabat, H. E., and Paterson, D. L. (2011). Escherichia coli O25bST131: a pandemic, multiresistant, community-associated strain. J. Antimicrob. Chemother. 66, 1-14. doi: 10.1093/jac/dkq415

Shibasaki, M., Komatsu, M., Sueyoshi, N., Maeda, M., Uchida, T., Yonezawa, H., et al. (2016). Community spread of extended-spectrum beta-lactamaseproducing bacteria detected in social insurance hospitals throughout Japan. J. Infect. Chemother. 22, 395-399. doi: 10.1016/j.jiac.2016.03.001

Takada, T., Kawarada, Y., Nimura, Y., Yoshida, M., Mayumi, T., Sekimoto, M., et al. (2007). Background: Tokyo Guidelines for the management of acute cholangitis and cholecystitis. J. Hepatobiliary. Pancreat. Surg. 14, 1-10. doi: 10.1007/s00534-006-1150-0

Takada, T., Strasberg, S. M., Solomkin, J. S., Pitt, H. A., Gomi, H., Yoshida, M., et al. (2013). TG13: updated Tokyo guidelines for the management of acute cholangitis and cholecystitis. J. Hepatobiliary Pancreat. Sci. 20, 1-7. doi: 10.1007/s00534-012-0566-y

Takeuchi, F., Sekizuka, T., Yamashita, A., Ogasawara, Y., Mizuta, K., and Kuroda, M. (2014). MePIC, metagenomic pathogen identification for clinical specimens. Jpn. J. Infect. Dis. 67, 62-65. doi: 10.7883/yoken.67.62

Tang, P., and Chiu, C. (2010). Metagenomics for the discovery of novel human viruses. Future Microbiol. 5, 177-189. doi: 10.2217/fmb.09.120

Tap, J., Mondot, S., Levenez, F., Pelletier, E., Caron, C., Furet, J. P., et al. (2009). Towards the human intestinal microbiota phylogenetic core. Environ. Microbiol. 11, 2574-2584. doi: 10.1111/j.1462-2920.2009.01982.x

van der Linden, W., and Sunzel, H. (1970). Early versus delayed operation for acute cholecystitis. A controlled clinical trial. Am. J. Surg. 120, 7-13. doi: 10.1016/S0002-9610(70)80133-7

Wang, C. H., Wu, C. Y., Yang, J. C., Lien, W. C., Wang, H. P., Liu, K. L., et al. (2016). Long-term outcomes of patients with acute cholecystitis after successful percutaneous cholecystostomy treatment and the risk factors for recurrence: a decade experience at a single center. PLOS ONE 11:e0148017. doi: 10.1371/journal.pone.0148017

Wells, J. E., Berr, F., Thomas, L. A., Dowling, R. H., and Hylemon, P. B. (2000). Isolation and characterization of cholic acid $7 \alpha$-dehydroxylating fecal bacteria from cholesterol gallstone patients. J. Hepatol. 32, 4-10. doi: 10.1016/S0168-8278(00)80183-X

Wu, T., Zhang, Z., Liu, B., Hou, D., Liang, Y., Zhang, J., et al. (2013). Gut microbiota dysbiosis and bacterial community assembly associated with cholesterol gallstones in large-scale study. BMC Genomics 14:669. doi: 10.1186/1471-2164-14-669

Zankari, E., Hasman, H., Cosentino, S., Vestergaard, M., Rasmussen, S., Lund, O., et al. (2012). Identification of acquired antimicrobial resistance genes. $J$. Antimicrob. Chemother. 67, 2640-2644. doi: 10.1093/jac/dks261

Conflict of Interest Statement: The authors declare that the research was conducted in the absence of any commercial or financial relationships that could be construed as a potential conflict of interest.

Copyright (C) 2017 Kujiraoka, Kuroda, Asai, Sekizuka, Kato, Watanabe, Matsukiyo, Saito, Ishii, Katada, Saida and Kusachi. This is an open-access article distributed under the terms of the Creative Commons Attribution License (CC BY). The use, distribution or reproduction in other forums is permitted, provided the original author(s) or licensor are credited and that the original publication in this journal is cited, in accordance with accepted academic practice. No use, distribution or reproduction is permitted which does not comply with these terms. 